\title{
Tic, culture, territoire : le trièdre du développement patrimonial
}

\section{Marie-Michèle Venturini}

\section{(2) OpenEdition}

1 Journals

Édition électronique

URL : http://journals.openedition.org/communicationorganisation/3481

DOI : 10.4000/communicationorganisation.3481

ISSN : $1775-3546$

Éditeur

Presses universitaires de Bordeaux

Édition imprimée

Date de publication : 1 octobre 2006

Pagination : 216-228

ISSN : 1168-5549

\section{Référence électronique}

Marie-Michèle Venturini, «Tic, culture, territoire : le trièdre du développement patrimonial »,

Communication et organisation [En ligne], 30 | 2006, mis en ligne le 19 décembre 2012, consulté le 20 avril 2019. URL : http://journals.openedition.org/communicationorganisation/3481 ; DOI : 10.4000/ communicationorganisation.3481

Ce document a été généré automatiquement le 20 avril 2019

(C) Presses universitaires de Bordeaux 


\title{
Tic, culture, territoire : le trièdre du développement patrimonial
}

\author{
Marie-Michèle Venturini
}

\section{Des frontières territoriales redessinées}

\section{Tic et territoire}

1 Si les Tic contractent les distances, ouvrent les frontières, réduisent les contraintes géographiques, elles nous proposent un espace virtuel, hors du temps et de l'espace physique, le cyberespace.

2 Ce territoire de l'intangible et de distances abolies, du moins en théorie, résulte de l'utilisation des technologies de l'information et de communication - et de l'action humaine. Il s'agit d'un espace virtuel au cœur d'Internet et des réseaux d'information, de télécommunication hauts débits et des serveurs de données disséminés sur la planète.

Cet espace virtuel, porte et fait s'éclore de nouvelles relations sociales, de nouveaux modes de création artistique et d'accès à la culture, de nouvelles façons de commercer, de se distraire, de se soigner, de travailler, bouleverse les relations de connexité entre les territoires, s'affranchit des contraintes de distances, repositionne le «local » face au "global». Les acteurs vivent, partagent ou subissent de nouvelles temporalités, complémentaires ou en décalage de leurs modes de vie présents, préfigurant les évolutions sociétales que l'on désigne par « société de l'information ».

Cette liberté relative modifie notre perception de l'espace, notre rapport aux autres et permet aux hommes de s'affranchir des contraintes d'éloignement et d'isolement géographique. Lorsque un acteur isolé ou membre d'un groupe se dote du minimum technologique requis et peut accéder à cette 'connectivité' réticulaire, il peut vivre l'instantanéité des échanges immatériels et des communications entre les personnes, quelle que soit sa localisation sur la planète.

5 Mais nous situerons notre propos à un niveau autre, celui de d'une synergie des ressources, des savoir-faire, des connaissances des hommes et des organisations, avec à la 
clef la mise en oeuvre d'un processus d'intelligence collective qui va probablement révolutionner notre société ${ }^{1}$.

\section{Une communauté de devenir à susciter}

6 L'attractivité territoriale suppose que la motivation d'une implantation future ne soit pas uniquement liée à une sensibilité à l'identique de celle des individus résidant. De cette distance peut apparaître ou naître un certain nombre de malentendus notamment culturels avec l'ensemble des acteurs locaux. De ce fait, l'intégration ne pourra s'effectuer. C'est dans ce sens qu'il nous semble nécessaire de re-considérer dans un premier temps que le territoire est habité par une communauté humaine et qu'il existe entre le territoire et ses ressources ce que nous pourrions nommer une "alliance culturelle » qui peut déboucher dans le meilleur des cas sur une « vocation culturelle ».

7 La «vocation culturelle " d'un territoire en fait dès lors un lieu singulier, unique, porteur à la fois de valeurs patrimoniales culturelles, de savoirs et de savoir-faire originaux, d'ambitions et de projets d'avenir. Le retour vers le local relevé, ici ou là, peut pleinement se nourrir au travers de la médiation patrimoniale.

\section{Un retour vers le local : la médiation patrimoniale}

8 En effet, l'histoire de notre civilisation nous permet de constater une évolution certaine du local vers le global puisque les sociétés ont mis toute leur énergie à lutter contre la parcellisation, à se rassembler, à développer les moyens de communication et les productions susceptibles d'être échangées. Cependant, aujourd'hui, nous assistons au processus inverse, c'est-à-dire que la question n'est plus de savoir si nous tendons vers l'émergence d'une culture mondiale, mais si les cultures locales continueront d'exister autrement qu'à l'état de produits folkloriques. Les processus d'universalisation des cultures se poursuivent avec une telle énergie que nous pourrions voir poindre une sorte d'asphyxie des cultures locales, à moins d'un ressaisissement et d'un immense effort collectif leur permettant de continuer à exister. Nous pouvons affirmer que bon nombre de pratiques et de traditions ont désormais disparu.

9 De plus, un patrimoine qui n'est pas valorisé ni même partagé peut selon nous disparaître. Il ne s'agit pas non plus qu'un groupe restreint et/ou élitiste se l'approprie. Il fait partie intégrante de la culture qui nous unit. Nous pouvons avancer que communiquer notre patrimoine équivaut à débattre de notre identité et de notre projet de devenir. C'est la raison pour laquelle les institutions européennes ont mis en place des actions de valorisation et d'éducation visant à rapprocher les pays européens autour d'actions pédagogiques communes comme par exemple les classes européennes du patrimoine, les itinéraires culturels, ou encore les opérations Enseignement sans frontières ... Ces opérations veulent relever d'une pédagogie active interdisciplinaire fondée sur le patrimoine culturel. Notre objectif est de ce fait d'approfondir la notion de médiation entre les acteurs et de s'interroger sur la dimension éthique de la diffusion du patrimoine, c'est-à-dire de faire reconnaître à tous un patrimoine culturel. 


\section{Un défi : patrimoine et développement}

10 Le patrimoine est un vecteur du développement local parce qu'il se compose de ressources locales et fait l'objet d'études et de recherches approfondies. «Il est doté de facteurs économiques et sociaux classiques ou génériques comme par exemple la capacité de travail, le capital mobilisable et les matières premières. Parmi celles ci, certaines peuvent être spécifiques au territoire ce qui permet d'expliquer la création d'activités nouvelles. Elles semblent dédiées à un usage et à un lieu et leur valorisation dépend d'une stratégie de territoire impulsée par une combinaison d'acteurs d'origine très diverse $»^{2}$. Le processus de développement correspond à la recherche de spécificités au sein desquels le patrimoine joue un rôle concurrentiel moteur. «Combinée à la fonction de prospective, elle révèle des potentiels de développement $\|^{3}$. La numérisation de données patrimoniales peut en cela aider à formuler la fonction de prospective à définir pour un territoire donné. C'est la posture adoptée par les tenants de l'intelligence territoriale qui fait largement appel aux possibilités offertes par l'appropriation des Tic.

\section{La numérisation, vecteur d'une politique d'innovation}

11 Au cours de ces vingt dernières années, les bases de données sur le patrimoine culturel national se sont fortement enrichies et les plus consultées, comme par exemple celles de la BNF ou encore celle du Musée du Louvre, ont été complétées par des banques d'images électroniques permettant d'associer les fiches textuelles aux images des oeuvres ou des monuments. Depuis 1996, un programme national (le Plan de numérisation des fonds iconographiques, sonores et audiovisuel) programme géré par la MRT (Mission de la Recherche et de la Technologie) de numérisation a en effet été mis en place.

12 La multiplicité des initiatives, individuelles et collectives, pour publier des ressources peut être considérée comme facteur de richesses culturelles et d'innovations, mais elle pose un défi en matière d'interopérabilité et d'accès notamment en ce qui concerne le fait de faciliter les échanges entre les utilisateurs et d'atteindre à une dimension universelle.

\section{La mise en valeur, le patrimoine spécifique d'une région}

Posée de la sorte, cette problématique de patrimoine et territoire verra donc se multiplier les propositions de découverte du patrimoine de proximité, contribuant ainsi à confirmer auprès du grand public l'élargissement de la notion de patrimoine observé depuis près d'un demi-siècle. Ce sera l'occasion, pour les acteurs locaux, de mettre en valeur le patrimoine spécifique d'une région, qu'il soit déjà pleinement légitimé par les procédures réglementaires de protection ou en phase d'émergence.

$14 \mathrm{Au}$ fond, la question centrale posée par cette relation pourrait être : «Qu'est-ce qui fait bien commun pour les gens qui vivent ici ? $»^{4}$. Corollairement, nous pouvons nous demander également : «Quel élément patrimonial est à même de représenter le territoire d'ici pour les gens qui viennent ou qui le découvrent d'ailleurs? ", tant le patrimoine - il 
suffit de regarder les logotypes des collectivités territoriales ou les panneaux qui bordent les autoroutes - constitue bien souvent l'emblème, voire l'icône d'un territoire.

Nous privilégions ici trois approches qui selon nous permettent de mettre en évidence le lien existant entre le patrimoine et le territoire.

- La première approche, scientifique, consiste à mettre en valeur les éléments patrimoniaux singuliers d'un territoire, ceux-là même qui fondent son originalité et le rendent différent et spécifique.

- La deuxième approche, plus sociale, privilégie quant à elle les questions de société auxquelles le patrimoine est immanquablement lié.

- Enfin, une approche nouvelle de la politique culturelle va naître des réaménagements territoriaux issus des lois sur la création des « Pays » et des structures intercommunales.

Nous aborderons dans les lignes suivantes le rôle des collectivités territoriales et de leur implication dans les processus de patrimonialisation.

\section{La responsabilité des collectivités territoriales}

Les collectivités locales prennent de plus en plus d'initiatives en matière de numérisation de leur patrimoine culturel et scientifique comme c'est le cas notamment de certaines bibliothèques municipales, d'Archives départementales ou de sites Internet. Leur objectif principal est d'offrir de nouveaux outils d'information et de recherche, à la fois pour la connaissance et l'enseignement mais également pour le tourisme culturel. Plusieurs grands programmes de numérisation et de valorisation des ressources ont été engagés ou sont en projet dans les régions comme par exemple celui des archives départementales de la Corse, celui des journaux locaux de Corse du Sud des XIX et XX ${ }^{e}$ siècles, ou encore le projet de création d'une médiathèque culturelle de la Corse en tant qu'outil multimédia de visualisation et d'aide à l'analyse des phénomènes culturels. Certains sont destinés à des institutions muséales, d'autres au domaine de l'enseignement et de la recherche.

Ces programmes apportent aux institutions et aux acteurs locaux des moyens matériels et humains nouveaux pour la production de contenus culturels sur les réseaux et soutiennent le développement économique régional à travers de nouveaux services et peuvent devenir peut- être des facteurs de développement du territoire.

\section{La numérisation du patrimoine, facteur de développement}

19 La numérisation permet d'envisager « des perspectives nouvelles de préservation, mais aussi d'étude et de mise à disposition des patrimoines " ${ }^{5}$ afin de favoriser leur appropriation et leur partage. Que ce soit les patrimoines immatériels ou les patrimoines matériels la numérisation peut être à la fois un outil d'aide à la sauvegarde, mais aussi un outil essentiel de diffusion, d'accès médiatisé, de partage, et d'enrichissement dans leur valorisation.

Nous pouvons envisager une politique ambitieuse et volontariste de numérisation du patrimoine en alliant les technologies, la mise en commun des ressources et surtout des compétences, des volontés et des moyens. "La numérisation du patrimoine est donc un enjeu à la fois culturel, touristique, éducatif et économique ${ }^{6}$. 
21 Ceci étant, les enjeux culturels, touristiques et économiques de la valorisation multimédia des ressources régionales, impliquent la coordination d'une stratégie régionale volontariste sur la numérisation du patrimoine en partenariat avec les grands acteurs touristiques et patrimoniaux publics et privés. Nous rejoignons à nouveau la posture qualifiée d'intelligence territoriale.

«Les technologies de l'information et de la communication ouvrent un nouvel espace culturel $»^{7}$, qui peut concourir fortement à l'aménagement du territoire et à la politique culturelle de l'Etat en région. En effet, le numérique permet d'imaginer de nouveaux modes d'accès à la culture et à la connaissance, en zone rurale et urbaine, indépendamment de la géographie. Il permet de créer, seul ou en réseau, et de favoriser une création pluridisciplinaire qui entretient des liens forts avec les entreprises culturelles comme par exemple le design, le multimédia, l'audiovisuel sur l'Internet... Ces industries culturelles numériques sont créatrices d'emplois et semblent attirer les jeunes générations au vu du développement des filières d'enseignement s'y rapportant.

Le développement des technologies numériques sur le territoire doit donc être également mis au service de la culture.

Cinq points essentiels selon nous émergent de cette proposition:

- La constitution de la mémoire numérique du territoire grâce notamment à la numérisation du patrimoine.

- La connexion des lieux culturels dans le but de développer des extranets culturels territoriaux.

- L'apprentissage des arts appliqués numériques au sein d'écoles d'art.

- Mais également, la production de culture par les acteurs locaux eux-mêmes afin de favoriser la création et le développement d'industries culturelles numériques.

- Enfin, le développement de structures administratives culturelles numériques.

\section{Les nouveaux territoires culturels ou de la culture}

De nos jours, nous pouvons constater que de plus en plus d'institutions entreprennent la numérisation de leurs fonds ou collections. Pour faciliter l'explication de cet engouement pour la numérisation du patrimoine culturel nous souhaitons revenir sur la définition du terme « numérisation ».

Le Dictionnaire encyclopédique de l'information et de la documentation ${ }^{8}$ définit la numérisation comme " un procédé électronique de production de signaux électriques numériques soit à partir d'un document ou d'un objet physique, soit à partir d'un signal électrique analogique. Le fichier numérique permet des traitements informatiques et, notamment, la réplication illimitée et sans perte de qualité indispensable à l'archivage et à la diffusion des documents", et détaille ensuite les différentes techniques de numérisation en fonction des objets pris en compte (feuillets, documents reliés, photographies, objets en 3D, sons ...).

De même, la Mission de la Recherche et de la Technologie (MRT) définit la numérisation comme « la codification numérique des intensités lumineuses et de la colorimétrie d'un document. La représentation numérique d'un texte peut se faire selon deux modalités :

- Le texte est considéré comme une image qui permet de rendre seulement la forme des caractères, il est alors représenté sur un mode photographique. Ce type de document est obtenu par numérisation directe du document (mode image). 
- Chaque caractère a sa représentation unique sous forme numérique, ce type de document en mode caractère est obtenu soit par saisie directe par des outils de type traitement de texte, soit par reconnaissance optique de caractères à partir d'un document en mode image $»^{9}$.

A partir de ces définitions, entre autres, nous pouvons penser que la numérisation est la transformation du format d'origine d'un document en format électronique.

De ce fait, la numérisation du patrimoine culturel peut participer et encourager la création de savoirs et de savoir-faire en vue de les partager.

Nous rejoignons volontiers l'idée que «les technologies multimédias offrent bien plus qu'un support médiatique à exploiter économiquement. Elles apportent un nouveau système d'échange, de solidarité, d'éducation et de partage de la connaissance du patrimoine culturel français, européen et mondial $»^{10}$. Plusieurs programmes de numérisation ont d'ailleurs été mis en place en France, comme par exemple celui du Ministère de la Culture et de la Communication, entre autres, permettant ainsi qu public de s'approprier les documents numérisés tout en facilitant le travail des chercheurs. Ce sont là autant d'atouts nouveaux à exploiter.

De même, la numérisation ouvre de plus en plus l'accès au patrimoine culturel puisque certaines bibliothèques numériques consultables via l'Internet permettant ainsi la possibilité de visiter virtuellement des expositions. Cela nous permet de percevoir tout l'intérêt que peut représenter la numérisation du patrimoine culturel pour les institutions et par là même pour le territoire qui les accueille.

\section{Bibliothèques, musées : des dispositifs au service du patrimoine culturel}

Jean Guattégno écrit : «Le XIX ${ }^{\mathrm{e}}$ siècle nous avait légué des bâtiments imposants, moitié musée, moitié bibliothèque, la bibliothèque en général à l'étage, équipée de rayons inaccessibles sans ces merveilleuses échelles de bois que nous avons connues ${ }^{11}$

Aujourd'hui, nous assistons au passage de la bibliothèque à la médiathèque via le processus de numérisation du patrimoine culturel. La bibliothèque en tant « qu'outil de la démocratisation culturelle " ${ }^{12}$ participe désormais en plus à la construction de l'image urbaine, c'est-à-dire que de plus en plus de villes modernisent et valorisent leur bibliothèque et par là même l'image culturelle de leur cité, et nous constatons la dimension à la fois sociale et culturelle de la médiation qui créé notre appartenance et notre citoyenneté.

«Les musées suivent une évolution parallèle » et deviennent alors « un formidable outil de notoriété et de communication, dans le contexte d'explosion du marché de l'art qu'on connu les années $1980 »^{13}$, année par excellence du patrimoine.

\section{L'intelligence territoriale et le patrimoine : une voie et des voix pour constituer la mémoire}

Dans nos propos précédents, nous avons établi la relation à imaginer entre le territoire et son développement par la patrimonialisation de ses constituants culturels. Nous avons évoqué que cette posture relevait d'un processus, l'intelligence territoriale. Nous emprunterons la définition de l'intelligence territoriale à Yann Bertacchini (2004) en tant 
«processus informationnel et anthropologique, régulier et continu, initié par des acteurs locaux physiquement présents et/ou distants qui s'approprient les ressources d'un espace en mobilisant puis en transformant l'énergie du système territorial en capacité de projet. De ce fait, l'intelligence territoriale peut tout à fait être assimilée à la territorialité qui résulte du phénomène d'appropriation des ressources d'un territoire puis aux transferts des compétences entre des catégories d'acteurs locaux de culture différents » ${ }^{14}$.

De plus, nous vivons non pas une identité unique, mais des identités multiples et croisées. C'est pourquoi la dimension territoriale a de l'importance pour penser le patrimoine. Les possibilités d'activité et d'identification qui nous entourent ne sont pas aléatoires. Elles sont le fruit de la volonté des militants associatifs, de sollicitations institutionnelles, de l'histoire spécifique d'une zone géographique. Les données de notre environnement immédiat social, économique, culturel, sont de plus en plus informatisées. Et de plus en plus, les informatisations successives mettent en évidence l'ensemble des possibilités d'action et d'identification réunies dans un lieu. C'est le cas par exemple du portail d'une mairie sur Internet. Enfin, ces informatisations successives mettent en interconnexion des activités internes à un groupe avec des activités associées. Ces actions soulignent la multiplicité des intentions et peuvent brouiller aussi la lecture d'un territoire.

Nous pouvons alors nous demander comment la numérisation du patrimoine régional participe-t-elle de l'intelligence territoriale ? On pourrait proposer en ce sens une réflexion systématique sur la numérisation du patrimoine de la région, s'appuyant sur la diversité de ce qui est travaillé aujourd'hui au sein d'un département par exemple. Nous nous intéresserons plus particulièrement à la question de la production de l'information c'est-à-dire au recensement et à la description des éléments patrimoniaux. Nous pensons que si le premier maillon à savoir la prise en compte du patrimoine tangible, est défaillant, c'est toute la chaîne de l'information jusqu'à la numérisation qui est faussée.

La numérisation permet de nouvelles formes d'accès aux archives et au patrimoine public qui suscitent le renouveau de pratiques anciennes, par exemple, la valorisation et la mise en ligne d'archives personnelles ou associatives.

La numérisation du patrimoine et sa mise à disposition répondent à la demande croissante de la population de constituer collectivement la mémoire des groupes et des territoires, socle des cultures et des pratiques culturelles.

Les programmes et les méthodes techniques en faveur de la numérisation des fonds patrimoniaux du ministère de la culture évoqués dans les lignes précédentes s'étendent progressivement à l'ensemble des territoires.

\section{Conclusion}

41 L'échange, la mutualisation des savoirs et savoir-faire, la collaboration, le partage des ressources, sont autant de mot clé désignant la volonté qui se dégage aujourd'hui, face à la montée en puissance du global, pour favoriser le développement de la culture locale. "En associant les acteurs et la communauté d'un territoire à la mutualisation des informations et à leur exploitation coopérative, l'intelligence territoriale a pour but d'améliorer dans un processus interactif, itératif et prospectif, leur compréhension de la structure et des dynamiques du territoire ainsi que leur maitrise collective du développement territorial. ${ }^{15}$ L'utilisation des TIC est donc un volet majeur de l'application des processus d'intelligence territoriale. Ce paradigme semble représenter la clé stratégique de nombreux enjeux pour les projets de 
développement dans le domaine culturel, entre autres, pour une région. Nous considérons les "process» d'intelligence territoriale comme l'approche pragmatique pertinente pour la mise en réseaux des acteurs notamment dans le partage des connaissances. L'entrée dans la société de l'information est un mouvement inéluctable dès lors que les connexions sont démocratisées, mais n'est pas une fin en soi. Elle amène à l'élaboration de nouveaux rapports des acteurs, une nouvelle configuration des collectivités dans un "construit» commun. Le paysage numérique est un construit commun des populations de décideurs engagés dans des processus collectifs. Dans la recherche d'un maillage des compétences territoriales pertinent, une clé importante réside dans la construction d'un maillage numérique adéquat, support de travail et d'échanges d'informations maîtrisé. La question du maillage numérique peut sembler éminemment complexe. Elle ne sera toujours malgré tout possible que par la mise en évidence d'un besoin humain dans une dynamique de concertation et d'élaboration partagée. Le numérique vient solutionner les difficultés réelles en même temps qu'il est un construit d'un collectif et donc fruit d'une culture partagée. Internet peut faire surgir la représentation de nouvelles cartes, celles des territoires de projets ou des régions culturelles.

\section{BIBLIOGRAPHIE}

Aubin, S., La numérisation du patrimoine culturel, Conservatoire des arts et Métiers, 2003.

Auriac. F et Brunet R. (dir.), Fayard, Roncayolo M., La ville et ses territoires, Gallimard, Folio essais, 1990.

Badie. B, La fin des territoires, Paris, Ed. Fayard, 1995.

Bertacchini, Y. Intelligence territoriale, - volet 2 - Mesurer la distance; Penser la durée ; mémoriser le virtuel, Coll. Les Etic, p. 275.

Bres, S., Jolion, J-M, Lebourgeois, F., Traitement et analyse des images numériques, Paris, Hermès, $2003,416 \mathrm{p}$.

Brunet.R, Dollfus.O, Mondes nouveaux, Géographie universelle, tome 1, Hachette-RECLUS, 1990.

Cacaly, S., (Dir.), Dictionnaire encyclopédique de l'information et de la documentation, Paris, Nathan, 2001.

Coüasnon, B., Dalbéra, J-P, Empotz, H., « Numérisation et patrimoine », in Document numérique, $\mathrm{N}^{\circ}$ spécial, vol. 7, n³-4, 2003.

Dalbéra, J-P., « Recherche et numérisation du patrimoine culturel », in Actes Ichim 2003, Paris, Ecole du Louvre, 8-12 sept. , 2003.

Ferrier.J.P., Antée 1, la géographie çà sert d'abord à parler du territoire, éditions Edisud, 1984.

Le Berre M., (1992), «Territoire », in A.S.Bailly et D. Pumain, (dir.) Encyclopédie de la Géographie, Paris, Economica, 1992.

Communication et organisation, $30 \mid 2006$ 
Lévy, P., L'intelligence collective. Pour une anthropologie du cyberespace, Paris, La Découverte, 1994.

Jokilheto, Jukka, Lusenet, Yola de, Gertz, Janet, " La conservation à l'ère du numérique » in Actes des IVème Journées Internationales de l'ARSAG, Paris, 27-30 mai 2002, Association pour la recherche scientifique sur les arts graphiques, 2002.

Ory-Lavollee, B., La diffusion numérique du patrimoine, dimension de la politique culturelle, rapport à Madame la Ministre de la culture et de la communication, janvier 2002.

Papy, F. (dir.), Les bibliothèques numériques, Paris, Hermès-Lavoisier, 2005, 224 p.

Perrot, X., « Patrimoine et culture numérique » in Actes de la conférences ICHIM, Berlin, 31 Août - 2 sept. 2004.

Robinson, M., Dictionnaire de terminologie numérique, Paris, Ellipses, 2004. 704 p.

Souchier, E., Jeanneret, Y., Le Marrec, J., Lire, écrire, récrire. Objets, signes et pratiques des médias informatisés, Paris, BPI/Centre Pompidou, 2003, 352 p.

Stasse, F., Rapport au ministre de la culture et de la communication sur l'accès aux œuvres numériques conservées par les bibliothèques publiques, Paris, avril 2005, 2005, 16 p.

Tayeb, M., «La numérisation du patrimoine culturel », in Culture et Recherche, ${ }^{\circ} 100$, janvier-mars 2004.

Guide des bonnes pratiques, version 1.3, (2004), dans le cadre du projet Minerva, par le groupe de travail Minerva ${ }^{\circ} 6$, identification des bonnes pratiques et des centres de compétences, http:// www.numerique.culture.fr/fr/europe/documents/bonnes_pratiques.pdf.

Normes et documents numériques : quels changements ? (1999-2000), in Solaris, dossier n6, déc.1999janv. 2000 http://www.info.unicaen.fr/bnum/jelec/Solaris/d06/

La préservation des documents numériques : solutions techniques, solutions politiques, Journée d'étude organisée par l'Association des directeurs et des personnels de direction des bibliothèques universitaires et de la documentation, à l'Université de Provence, Aix-Marseille 1, 14/09/2001 http://www-sv.cict.fr/adbu/actes_et_je/je2001/Insc_journee12.html

Numérisation et patrimoine, (2004), Premières rencontres, La Rochelle, 21-23 juin 2004, 2 tomes, 80 et $70 \mathrm{p}$.

\section{NOTES}

1. Levy, Pierre, L'intelligence collective ; pour une anthropologie du cyberespace, Paris, la Découverte, 1994.

2. Cf. http://iga.ujf.grenoble.fr/cermosem

3. Ibid.

4. http://www.vie-publique.fr/document-vp/journeepatrimoine.shtml

5. http://www.paris-region.com/upload/document/D168.pdf

6. Ibid.

7. http://www.memoirelocale.com/document/CIADT.html

8. Sous la direction de Serge CACALY, Paris : Nathan, p. 431-433, 2001.

9. Fiche de la Direction du Livre et de la Lecture : « La numérisation dans les bibliothèques ». URL : www.culture.fr/culture/mrt/numerisation/fr/dll/techn.htm

10. Aubin, Sophie, La numérisation du patrimoine culturel, Conservatoire des Arts et métiers, 2003. 
11. Jean Gattégno, in « Bibliothèques et lecture publique », Le débat, $\mathrm{n}^{\circ}$ 48, 1988, p. 93-102, p. 95. 3. Le rôle des collectivités locales.

12. Aubin, Sophie, Op.Cit.

13. Ibid.

14. Bertacchini, Yann, Intelligence territoriale, - volet 2 - Mesurer la distance; Penser la durée; mémoriser le virtuel, Coll. Les Etic, p. 275.

15. Girardot, Jean Jacques, «Intelligence territoriale et participation, in $3^{\text {èmes }}$ rencontres « Tic et Territoire : quels développements? », Lille, in ISDM n 16 , mai 2004.

\section{RÉSUMÉS}

Notre objectif à travers cet article est de démontrer l'importance de la numérisation du patrimoine culturel corse pour le développement local du territoire, tant au niveau de la valorisation de ses ressources que du partage des savoirs et savoir-faire locaux. A partir de définitions possibles $\mathrm{du}$ territoire, nous abordons son aménagement à l'aide des Tic et notamment de la numérisation du patrimoine culturel comme phénomène majeur du développement territorial. Nous nous positionnons dans l'optique d'une démarche ouverte d'intelligence territoriale. Nous situons notre réflexion au croisement des actions de patrimonialisation qui à notre sens consiste en la diffusion et la valorisation du patrimoine culturel.

Our objective with through this article is to show the importance of the digitalization of the Corsican inheritance for the local development of the territory, as well on the level of the valorization of its resources as of the division of the knowledge and local knowledge. From possible definitions of the territory, we approach his installation using the Tic and in particular of the digitalization of the cultural inheritance like major phenomenon of the territorial development. We position in the optics of an open step of territorial intelligence. We locate our reflexion at the crossing of the actions of patrimonialisation wich with our direction consists of the diffusion and the valorization of the cultural inheritance.

\section{INDEX}

Mots-clés : médiation, patrimoine culturel, Tic, territoire, numérisation, développement local

\section{AUTEUR}

\section{MARIE-MICHÈLE VENTURINI}

Marie-Michèle Venturini est enseignante à l'Université de Corse Pascal Paoli et responsable pédagogique de la filière Communication Europe et Méditerranée. Elle travaille sur la problématique « Hypermédias, patrimoine et territoire » au sein du CRESHS (Centre de Recherche et d'Etudes en Sciences Humaines et Sociales) de l'Université de Corse Pascal Paoli. Elle est également chercheur associé au laboratoire I3M de l'Université du Sud Toulon Var au 
sein de l'équipe dont la thématique de recherche est l'intelligence territoriale. Mail : mmpv@tiscali.fr 